\title{
Free Radicals: How Do We Stand Them? Anaerobic and Aerobic Free Radical (Chain) Reactions Involved in the Use of Fluorogenic Probes and in Biological Systems
}

\author{
Stefan I. Liochev
}

Durham, N.C., USA

\section{Key Words}

Superoxide dismutase · Free radicals · Reactive oxygen species · Superoxide reductase · Superoxide

\begin{abstract}
Biologically significant conclusions have been based on the use of fluorogenic and luminogenic probes for the detection of reactive species. The basic mechanisms of the processes involved have not been satisfactorily elucidated. In the present work, the mechanism of the enzyme and photosensitized oxidation of $\mathrm{NAD}(\mathrm{P}) \mathrm{H}$ by resorufin is analyzed and appears to involve both aerobic and anaerobic free radical chain reactions. There are two major fallouts of this analysis. Many of the conclusions about the participation of radicals based on the use of probes such as resorufin and Amplex red need reevaluation. It is also concluded that anaerobic free radical reactions may be biologically significant, and the possible existence of enzymatic systems to eliminate certain free radicals is discussed.

(c) 2014 S. Karger AG, Basel
\end{abstract}

\section{Introduction}

A number of publications involve the use of fluorogenic and luminogenic probes, such as fluorescein, dihydrorhodamine, lucigenin, etc., for the extracellular, intracellular and even intraorganellar measurement of reactive oxygen species (ROS). A number of publications warn about the inherent pitfalls due to the complexity of the reactions involved [1-6]. The authors of critical publications advise that while a given probe may be unsuitable to measure a particular species, it still may be suitable for other species and that the problems facing the use of a particular probe can be avoided by using a suitable probe for the intended purpose.

While being sympathetic to the views of the critics, this author's opinion is more skeptical than the views of even the fiercest of these critics. My view is that the intracellular measurement of species such as ROS using EPR, fluorescence, or luminescence should be supported by correct analysis of observations made by methods independent of the use of such probes.

Amplex red and its fluorogenic oxidation product resorufin (RSF) have been and still are among the most often used such probes, yet they are also among the most controversial [3]. The questionable view that manganese superoxide dismutase (MnSOD) is a major intracellular

\begin{tabular}{ll}
\hline KARGER & $\begin{array}{l}\text { ○ 2013 S. Karger AG, Basel } \\
1011-7571 / 13 / 0233-0195 \$ 38.00 / 0 \quad \text { Karger }\end{array}$ \\
E-Mail karger@karger.com & $\begin{array}{l}\text { This is an Open Access article licensed under the terms of the } \\
\text { Creative Commons Attribution-NonCommercial 3.0 Un- } \\
\text { ported license (CC BY-NC) (www.karger.com/OA-license), } \\
\text { applicable to the online version of the article only. Distribu- } \\
\text { tion permitted for non-commercial purposes only. }\end{array}$
\end{tabular}

Stefan I. Liochev

E-Mailliochev@yahoo.com 
source of hydrogen peroxide $\left(\mathrm{H}_{2} \mathrm{O}_{2}\right)$ and that overproduction of MnSOD increases the cellular production of $\mathrm{H}_{2} \mathrm{O}_{2}$ [7] are based on experiments utilizing these probes. Therefore, revisiting the mechanism of the processes these probes are involved in seems worthwhile.

In the first part of this paper a critical analysis of the experimental results described in two papers $[5,6]$ reveals that, even in vitro, the real mechanism of the 1-electron reduction of RSF by $\mathrm{NAD}(\mathrm{P}) \mathrm{H}$ is far from simple. The scheme here arrived at suggests the existence of coupled aerobic and anaerobic chain processes. Aerobically, the anaerobic chain process is replaced by a superoxide $\left(\mathrm{O}_{\overline{2}}^{\overline{2}}\right)$ dependent chain process. In the presence of other reactants, and certainly intracellularly, the complexity of the processes involved excludes any meaningful conclusions. The implications for the applicability of such probes for detection of ROS are also discussed.

The existence of an anaerobic chain process in the case of the RSF photocatalyzed oxidations suggests that the biological significance of anaerobic biological free radical reactions has been underestimated. Therefore, the second part of this review focuses on the oxygen-independent production and toxicity of free radicals, the protective systems employed by cells to counteract that toxicity, and the possible mechanisms of action of such systems.

\section{Mechanistic and Kinetic Considerations}

\section{Analysis of Two Mechanisms Proposed to Explain the RSF-Dependent NAD $(P) H$ Oxidation \\ Mechanism 1}

Dutton et al. [5] studied the oxidation of NADPH in the presence of both NADPH-cytochrome P450 reductase (PR) and RSF under aerobic conditions and reported a number of interesting observations and conclusions, including: (a) that the ratio between the NADPH and $\mathrm{O}_{2}$ consumed and the $\mathrm{H}_{2} \mathrm{O}_{2}$ formed was $1: 1: 1$; (b) that the rate of RSF reduction in the presence of PR and NADPH was comparable to the rate of $\mathrm{RSFH}_{2}$ oxidation seen when NADPH was exhausted, and SOD dramatically slowed that oxidation; SOD also increased the rate of RSF reduction when NADPH and PR were both present, and (c) that both the rate and the extent of RSF reduction increased with increases in [PR]. However, when the $\mathrm{O}_{2}$ consumption was measured in a similar situation, only the rate but not the extent of $\mathrm{O}_{2}$ consumption increased with increases in [PR]. In the case of the $\mathrm{O}_{2}$ consumption experiment, but not in the RSF reduction experiment, the limit reached was due to exhaustion of NADPH.
The mechanism they propose is essentially as follows:

(1) (PR-mediated) $\mathrm{NADH}+\mathrm{RSF}+\mathrm{H}^{+} \leftrightarrows \mathrm{NAD}^{+}+$ $\mathrm{RSFH}_{2}$ (fast)

(2) $\mathrm{RSFH}_{2}+\mathrm{O}_{2} \leftrightarrows \mathrm{RSF}^{-}+\mathrm{O}_{\dot{2}}^{\overline{2}}+2 \mathrm{H}^{+}$(very slow)

(3) $\mathrm{RSF}^{-}+\mathrm{O}_{2} \leftrightarrows \mathrm{RSF}+\mathrm{O}_{\overline{2}}^{-}$(fast)

(4) $\mathrm{RSFH}_{2}+\mathrm{O}_{\overline{2}}^{\bar{\Sigma}} \leftrightarrows \mathrm{RSF}^{\overline{-}}+\mathrm{H}_{2} \mathrm{O}_{2}$ (fast)

To avoid confusion it should be noted that the remarks in parentheses after each reaction refer to rates (not rate constants!) under the experimental conditions used.

$\mathrm{PR}$ is reduced by NADPH and subsequently in two 1-electron steps it reduces RSF to $\mathrm{RSFH}_{2}$. The resulting $\mathrm{RSFH}_{2}$ then reacts very slowly with $\mathrm{O}_{2}$ to form $\mathrm{RSF}^{-}$and $\mathrm{O}_{\overline{2}}^{\overline{2}}$ and initiates a chain reaction whose propagation steps consist of oxidation of $\mathrm{RSFH}_{2}$ by $\mathrm{O}_{\overline{2}}^{\overline{2}}$ and of $\mathrm{RSF}^{-}$by $\mathrm{O}_{2}$.

Despite the possibility of chain reaction(s) propagated by reactions 3 and 4 , the process this mechanism describes is a really nonchain one. Thus, the chain process of $\mathrm{RSFH}_{2}$ autooxidation serves only to regenerate RSF and then to be reduced by PR. Hence, the rate of $\mathrm{RSFH}_{2}$ formation is the same as the rate of $\mathrm{RSFH}_{2}$ disappearance. Since the chain length $=$ the rate of the entire process/the rate of the (PR-mediated) initiation reaction - 1 , and since in this mechanism that ratio is 1 , the chain length of the process is 0 . This is in fact a circular process of reduction of RSF to $\mathrm{RSFH}_{2}$ followed by an equally fast opposite process of oxidation of $\mathrm{RSFH}_{2}$ to RSF and $\mathrm{H}_{2} \mathrm{O}_{2}$. SOD acts by inhibiting $\mathrm{RSFH}_{2}$ oxidation, depriving PR of its substrate. According to this mechanism, $\mathrm{O}_{\overline{2}}^{\overline{2}}$ can only be made during the chain process of $\mathrm{RSFH}_{2}$ autooxidation.

Despite its elegance and simplicity, this mechanism is likely to be an oversimplification. Thus, an oxidation of $\mathrm{NADH}$ by the radical product of dichlorofluorescein oxidation has been reported [4]. Hence, it is likely that, in addition to reaction $3, \mathrm{RSF}^{-}$also disappears as in reaction 5:

(5) $\mathrm{RSF}^{-}+\mathrm{NADPH}+\mathrm{H}^{+} \leftrightarrows \mathrm{RSFH}_{2}+\mathrm{NADP}^{\bullet}$ (fast, but how fast?)

This should, aerobically, be followed by reaction 6:

(6) $\mathrm{NADP}^{\cdot}+\mathrm{O}_{2} \leftrightarrows \mathrm{NADP}^{+}+\mathrm{O}_{\dot{2}}^{-}$(fast)

Then, $\mathrm{O}_{\overline{2}}^{\overline{2}}$ oxidizes another $\mathrm{RSFH}_{2}$, as in reaction 4 .

Reactions 5 and 6 will not change the stoichiometry but may allow some more accumulation of $\mathrm{RSFH}_{2}$ aerobically, as actually observed.

\section{Mechanism 2}

A recent paper [6] reported that the fluorogenic RSF causes light-dependent oxidation of NADH.

They propose that after the formation of the excited state of RSF (RSF*) the mechanism is as follows:

(7) $\mathrm{RSF}^{*}+\mathrm{NADH} \leftrightarrows \mathrm{RSF}^{-}+\mathrm{NAD}^{*}+\mathrm{H}^{+}$ 
(6) $\mathrm{NAD}^{\bullet}+\mathrm{O}_{2} \leftrightarrows \mathrm{NAD}^{+}+\mathrm{O}_{\dot{2}}^{-}$

(3) $\mathrm{RSF}^{-}+\mathrm{O}_{2} \leftrightarrows \mathrm{RSF}+\mathrm{O}_{2}^{-}$

After that, at $\mathrm{pH} 7.4, \mathrm{O}_{2}^{-}$is protonated, to $\mathrm{HO}_{2}$ which starts a chain reaction by oxidizing $\mathrm{NADH}$, followed by reaction 6 and then by another protonation of the resulting $\mathrm{O}_{\dot{2}}^{\overline{2}}$.

While the experiments in that paper [6] are well performed, it needs to be said that an $\mathrm{HO}_{2}$-dependent chain process of oxidation of NADH should not play a significant role for two reasons:

- The oxidation of $\mathrm{NAD}(\mathrm{P}) \mathrm{H}$ by $\mathrm{O}_{\overline{2}}^{-}$at $\mathrm{pH} 7.4$ can only be seen at very substantial fluxes of $\mathrm{O}_{\dot{2}}^{-}$because $\mathrm{HO}_{2}$ is only a moderately fast oxidant of $\mathrm{NAD}(\mathrm{P}) \mathrm{H}$ and further since, at that $\mathrm{pH}$, there is approximately 1,000 times less $\mathrm{HO}_{2}$ than $\mathrm{O}_{2}^{-}$. That is why lactate dehydrogenase [8] or vanadate [9] has been seen to catalyze aerobic NAD $(\mathrm{P}) \mathrm{H}$ oxidation by chain mechanisms at physiological $\mathrm{pH}$.

- As is clearly evident from figure 4 of that study [6], which illustrates the photoreduction of RSF and the photooxidation of NADH under aerobic as well as under anaerobic conditions, the anaerobic photooxidation of NADH sensitized by RSF is at least as fast as the aerobic one. This leads to the following paradox: if reaction 7 is fast, then the production of $\mathrm{RSF}^{-}$and of NAD ${ }^{\circ}$ is fast as well. Then, if a chain reaction such as the $\mathrm{HO}_{2}$-dependent oxidation of NADH was involved, the rate seen aerobically would have to be much higher than the one seen anaerobically, but it is not! On the other hand, if a chain reaction was not involved, the aerobic and anaerobic rates should be practically the same, but SOD should not inhibit! However, in fact, SOD was seen by the authors to inhibit the $\mathrm{O}_{2}$ consumption close to $100 \%$, and of course this means that the oxidation of $\mathrm{NADH}$ was also inhibited close to $100 \%$ by SOD.

The only possible conclusion explaining this paradox seems to be that reaction 7 , and therefore the production of NAD and $\mathrm{RSF}^{-}$, is very slow and that SOD inhibits a chain reaction in which $\mathrm{O}_{2}^{\overline{2}}$ participates in its own regeneration. If this is the case, why is the anaerobic photooxidation of NADH as fast as the aerobic one? Obviously, because of an anaerobic chain reaction.

\section{Anaerobic Chain Reaction}

Consider the following reactions:

(7) $\mathrm{RSF}^{*}+\mathrm{NADH} \leftrightarrows \mathrm{RSF}^{-}+\mathrm{NAD}^{*}+\mathrm{H}^{+}$(very slow)

(5) $\mathrm{RSF}^{-}+\mathrm{NADH}+\mathrm{H}^{+} \leftrightarrows \mathrm{RSFH}_{2}+\mathrm{NAD}^{\cdot}$ (fast)

(8) $\mathrm{NAD}^{*}+\mathrm{RSF} \leftrightarrows \mathrm{NAD}^{+}+\mathrm{RSF}^{-}$(fast)

(9) $\mathrm{RSF}^{-}+\mathrm{RSF}^{-}+2 \mathrm{H}^{+} \leftrightarrows \mathrm{RSF}+\mathrm{RSFH}_{2}$ (not significant)
(10) $\mathrm{NAD}^{\bullet}+\mathrm{NAD}^{\bullet} \leftrightarrows(\mathrm{NAD})_{2}$ (not significant)

(11) $\mathrm{NAD}^{\cdot}+\mathrm{RSF}^{-}+2 \mathrm{H}^{+} \leftrightarrows \mathrm{NAD}^{+}+\mathrm{RSFH}_{2}$ (not significant)

Reaction 7 is now recognized as a very slow (perhaps due to extremely low [RSF*]) initiation reaction and each of the two radicals that are products of this reaction starts a chain propagated by reactions 5 and 8 . Reactions 9-11 are included in order to discuss to what extent they are chain breaking and/or significant. This scheme is in agreement with the observed stoichiometry of the process.

The arguments that favor the significance of reactions 5 and 8 include but are not limited to: (a) they explain the anaerobic results of Zhao et al. [6]; (b) NADH has been shown to react with the radical product of dichlorofluorescein [4]; (c) Zhao et al. [6] detected $\mathrm{RSF}^{-}$by EPR anaerobically at high $\mathrm{pH}$, but not at $\mathrm{pH} 7.4$, and concluded that, since at high $\mathrm{pH}$ it is likely to be a dianion, its oxidizing potency is lost at such $\mathrm{pH}$, and (d) NAD ${ }^{\circ}$ is known to be involved in comparatively fast reductions [10].

Reactions such as 9-11 are usually viewed as chain breaking and indeed they are. In this case, reaction 9 should be insignificant due to electrostatic repulsion. The rate constant of reaction 10 is quite high [11] but in the experimental conditions of Zhao et al. [6] [NAD*] will be very low. Moreover, $(\mathrm{NAD})_{2}$, thought to be a dead end, is not one really and can be involved in redox reactions that regenerate $\mathrm{NAD}^{+}$and/or NAD ${ }^{*}$ [10]. Similar arguments concern reaction 11 and in fact none of reactions 9-11 is really a dead end since they regenerate components involved in the overall process.

It is clear that the process, even anaerobically, is complex and not easily quantified. What is clear is that a long length chain reaction is involved in the anaerobic lightinduced reduction of RSF by NADH, which will end with the complete reduction of RSF. However, at a steady state in the presence of a supply of $\mathrm{NAD}(\mathrm{P}) \mathrm{H}$ and an oxidant of $\mathrm{RSFH}_{2}$, it may proceed for a long time.

\section{The Aerobic Chain Process}

After the generation of $\mathrm{RSF}^{-}$and NAD ${ }^{\circ}$ in the initiation reaction $7, \mathrm{RSF}^{-}$will react in the competing reactions 3 and 5 generating $\mathrm{O}_{\dot{2}}^{\overline{2}}$ and $\mathrm{RSF}$ or $\mathrm{RSFH}_{2}$ and NAD', respectively. The NAD generated in reactions 7 and 5 should react mostly with $\mathrm{O}_{2}$ as in reaction 6 due to its higher concentration in solutions at normal atmospheric pressure compared to that of RSF and the very high rate constant of reaction 6 [11]. Whether any NAD ${ }^{*}$ will react 
in the competing reaction 8 , which is so important anaerobically, will depend on the unknown rate constant.

Thus, aerobically, the main chain-propagating reactions will likely be:

(5) $\mathrm{RSF}^{-}+\mathrm{NADH}+\mathrm{H}^{+} \leftrightarrows \mathrm{RSFH}_{2}+\mathrm{NAD}^{\bullet}$ (fast)

(6) $\mathrm{NAD}^{\bullet}+\mathrm{O}_{2} \leftrightarrows \mathrm{NAD}^{+}+\mathrm{O}_{2}^{\overline{2}}$ (fast)

and

(4) $\mathrm{O}_{\overline{2}}^{\overline{2}}+\mathrm{RSFH}_{2} \leftrightarrows \mathrm{RSF}^{\overline{ }}+\mathrm{H}_{2} \mathrm{O}_{2}$ (fast)

(3r) (reverse) $\mathrm{O}_{\dot{2}}^{\overline{2}}+\mathrm{RSF} \leftrightarrows \mathrm{O}_{2}+\mathrm{RSF}^{-}$(fast)

This explains the effect of SOD. Reactions like reaction 3 (forward and reverse) in the case of similar compounds are known to occur, and various rate constants in the case of different such compounds are available [12]. Thus, in combination with reaction 5 , reaction 3 (reverse) may also play a role in the generation of $\mathrm{RSFH}_{2}$. Reaction 6 is well known and is extremely fast. Reaction 4 was established by Dutton et al. [5] and reaction 5 is established in this work. Some chain-breaking reactions like the oxidation of $\mathrm{RSF}^{-}$by $\mathrm{O}_{2}^{\overline{2}}$ may occur. This and the fact that reaction 5 is the only chain-propagating one that the anaerobic and aerobic chain processes share, and that it may be rate limiting, may explain the slightly higher rate of $\mathrm{NADH}$ photooxidation anaerobically [6].

It must be stressed that this analysis is for the cases of the reductase plus RSF and light- and RSF-sensitized oxidations of $\mathrm{NAD}(\mathrm{P}) \mathrm{H}$ under the conditions applied by Dutton et al. [5] and Zhao at al. [6]. In fact, intracellularly these mechanisms will be totally different depending on the availability of other reductants. For example, $\mathrm{RSF}^{-}$should react mostly with something other than just $\mathrm{NADH}$ or $\mathrm{O}_{2}$, again creating radicals, and it will be some of those radicals that may eventually oxidize NADH.

\section{Implications for the Intracellular Use of Fluorogenic and Luminogenic Probes for Measurement of ROS, RNS, and Other Species}

The implications in the case of Amplex red and RSF and likely for the similar fluorescein, as well as for other probes, are troubling. This conclusion agrees with opinions expressed in recent articles [2,3] where other aspects of these problems are analyzed and some hope for the development of better probes is provided.

In some rare cases, in well-defined systems or cells and providing the mechanism is sufficiently elucidated, these probes may be successfully used to gain some important knowledge. Thus, lucigenin should not be used as a general probe for $\mathrm{O}_{2}^{\overline{2}}$ since it itself can mediate the production of $\mathrm{O}_{2}^{\overline{2}}$ and in addition is being reduced and the reduced form reacts with various endogenous and extraneous reductants and oxidants [13]. Therefore, it cannot be used for evaluation of the intracellular efficiency of unspecific scavengers or the ability of redox-cycling agents to generate $\mathrm{O}_{2}^{-}$. We have reported several artifacts [13-15] in the cases of Mn-porphyrins as scavengers and paraquat as a redox cycling agent. However, despite its ability to produce $\mathrm{O}_{\dot{2}}^{\overline{2}}$ and be involved in other reactions, the lucigenin luminescence should be roughly proportional to the steady state $\left[\mathrm{O}_{\dot{2}}\right]$ achieved at various levels of SOD. Having this in mind and knowing the lucigenin redox reactions in vitro, we were able to estimate that, in the wildtype Escherichia coli cells, both cytoplasmic SODs scavenge about $90-95 \%$ of endogenous $\mathrm{O}_{2}^{\overline{2}}$ in competition with all other endogenous targets [14]. Undoubtedly, we could not have been certain of that if not for the substantial previous and subsequent work by us and others [14, $16,17]$ that established a proportional relationship between the intracellular decrease in SOD, the increase in $\left[\mathrm{O}_{\dot{2}}\right]$, and the increase in inactivation of iron-cluster-containing dehydratases. Using the lucigenin-dependent approach, we predicted also that desulfoferredoxin acts as a superoxide reductase (SOR) [18]. Perhaps in other comparatively defined situations, such as the case of SOD mutants of Caenorhabditis elegans [19], this method could be used, keeping in mind the caveats and pitfalls and that $C$. elegans is still enormously more complex than E. coli.

Concerning Amplex red and RSF, a paper claiming that MnSOD possesses peroxidase activity was recently published [20]. This conclusion was criticized [21] since the authors [20] did not take into account artifacts inherent to these probes in the analysis of their experimental results. In turn, the arguments expressed by Liochev and Fridovich [21] were questioned [22]. Clearly, as demonstrated by such debates, other reported conclusions dependent on experiments involving such probes may need to be reconsidered.

\section{Aerobic and Anaerobic Free Radical Reactions and Their Biological Significance}

The importance of reactions involving the oxygen-independent formation and disappearance of free radicals and especially of anaerobic free radical chain reactions is at best underestimated and often completely ignored in the field of free radical biology and medicine. This is regrettable since the concentration of oxygen in most of the cells of the organisms (bigger than C. elegans!) in vivo is approximately 10 times lower than that in vitro (cell cultures). 
RSF is a not an uncommon molecule. It is chemically similar to a variety of natural and artificial compounds. Even more so, this is the case for its reactivity, e.g. its capability to be involved in 1- as well 2-electron transfer processes leading to the formation of free radicals and other potentially harmful species. In this regard, the deduction that RSF can be a catalyst and/or a substrate of anaerobic chain reactions involving intracellular reductants like $\mathrm{NAD}(\mathrm{P}) \mathrm{H}$ is an important one whose potential biological significance should not be overlooked. Clearly, the possibility of oxygen-independent intracellular free radical chain reactions catalyzed by a variety of agents and involving a variety of radicals is suggested. Moreover, given the toxicity of free radicals in general, one wonders how the cells counteract it. The following discussion attempts to approach these problems.

\section{Superoxide Reductase}

SODs are incredibly efficient catalysts markedly decreasing the steady state level of $\mathrm{O}_{\dot{2}}$.

Free radical reactions should be going on anaerobically, induced by both endogenous and exogenous agents, e.g. RSF and other redox-cycling compounds. However, unlike the case of the aerobes that have SODs, anaerobes appear to not have a similar extremely effective system to deal directly with radicals. Is that the case? Indeed, no single enzyme or other system would be able to compete with the numerous targets for a variety of radicals. However, consider the following hypothesis which is analogous to the radical sink one [23]. Suppose that the endogenous and exogenous first- or second-generation radicals react with some abundant molecules (sinks), and that the resulting relatively less reactive radicals are the substrates of the hypothetical scavenging system(s). Which are the best candidates for such a hypothetical system?

SOR, which acts as an efficient electron donor: superoxide oxidoreductase $[24,25]$, is certainly a likely candidate. SOR indeed provides protection when the anaerobes are exposed to $\mathrm{O}_{2}$ [24]. Lombard et al. [25] noticed that the more or less strict anaerobes that have SOR only very occasionally encounter oxygen and that $\mathrm{Fe}$ of SOR was found to be exposed to the water environment, suggesting a lack of specificity. They proposed that SOR eliminates both $\mathrm{O}_{\dot{2}}^{-}$and its sources [25], but reasonable arguments [24] suggest that the latter is unlikely to be the biological purpose of SORs. Nonetheless, the implications of the present work suggest an additional function of SORs under both anaerobic and aerobic conditions.
One such function then could be to neutralize the radicals formed under strictly anaerobic conditions.

The following hypothetical scheme considers how SOR may operate as a general radical remover. In the reduced environment of the cell it is likely that the radicals formed $\left(\mathrm{R}^{*}\right)$ will oxidize the fully reduced forms of some abundant available reductants ( $\mathrm{SH}$ ).

Consider the scheme of such action:

(a) $\mathrm{R}^{\cdot}+\mathrm{SH} \leftrightarrows \mathrm{RH}+\mathrm{S}^{\cdot}$

(b) $\mathrm{SOR}-\mathrm{Fe}(\mathrm{III})+\mathrm{S} \cdot \leftrightarrows \mathrm{SOR}-\mathrm{Fe}(\mathrm{II})+\mathrm{S}$

(c) $\mathrm{SOR}-\mathrm{Fe}(\mathrm{II})+\mathrm{S}^{\cdot}+\mathrm{H}^{+} \leftrightarrows \mathrm{SOR}-\mathrm{Fe}(\mathrm{III})+\mathrm{SH}$

(d) SOR-Fe-(II) $+\mathrm{O}_{\dot{2}}^{\overline{2}}+2 \mathrm{H}^{+} \leftrightarrows$ SOR-Fe-(III) $+\mathrm{H}_{2} \mathrm{O}_{2}$

Reactions b and $c$ describe an anaerobic analog to the SOD reaction cycle although the $S^{*}$ radicals involved may be the same or produced from different sinks, while reaction $\mathrm{d}$ is pertinent only aerobically. In the absence of $\mathrm{O}_{2}$, SOR could thus act as a free radical disproportionase or radical:radical oxidoreductase. Certainly, a 1-electron transfer protein such as rubredoxin, which is considered a physiological reductant of SOR [26], can be the substrate and in this case SOR will act as a substrate:radical oxidoreductase (SROR). That these speculations are not purely theoretical is illustrated by the fact that reduced SOR may be efficiently oxidized by a radical formed by the 1-electron oxidation of Amplex red [27].

The reducing and nearly oxygen-deficient environment that the organisms having SORs usually inhabit makes it difficult for them to find final electron acceptors. In this case, the benefit of elimination of potentially toxic oxidizing radicals including superoxide by SORs is likely supplemented by the benefit of the latter serving as electron receptors. Typical aerobes do not usually suffer from lack of electron acceptors. For them superoxide is just a very toxic agent that needs to be eliminated and their superoxide dismutases deal with it by converting it to oxygen and $\mathrm{H}_{2} \mathrm{O}_{2}$. The latter two species are the electron acceptors of the oxidases and, respectively, peroxidases (including peroxiredoxins).

\section{Radical Sinks, SODs, SRORs, and Related Matters}

In a recent review, Winterbourn [23] raised awareness that while the biochemistry of free radicals and related oxidants is becoming well understood, the problem of how these reactions interplay in vivo is much less so and that kinetic considerations are a powerful tool to tackle this problem. A very important part of the review of Winterbourn [23] clarifies what constitutes antioxidant action. Specifically, a scavenging reaction is defined as pro- 
tective if it leads to a less damaging product. The review [23] and scheme 2 in it demonstrates how the free radical sink hypothesis explains the way this protection is achieved. Thus, the various radicals produced are scavenged by major sinks such as glutathione, ascorbate, and $\mathrm{O}_{2}$. The protection is due to the lower reactivity of the radicals of glutathione and ascorbate and because SOD scavenges the potentially dangerous $\mathrm{O}_{\overline{2}}$. Furthermore, the initial GS ${ }^{*}$ produced from GSH reacts with another GSH leading to the formation of GSSG ${ }^{\cdot-}$, which is rapidly autooxidized, and the resulting $\mathrm{O}_{\overline{2}}^{-}$is scavenged by SOD. The resulting GSSG should be then easily converted back to GSH by the glutathione reductase, while the ascorbyl radical is processed to yield ascorbate and dehydroascorbate. In addition, GS ${ }^{*}$ may be reduced by other electron donors or may react with $\mathrm{O}_{2}$ to yield GSOO ${ }^{*}$ which reacts with other sinks, as discussed by Wardman [28]. The Winterbourn [23] review thus gives a clear and broad description of the aerobic processes of protection.

The following text discusses points that the author feels are important but remained underanalyzed or not discussed at all. One such point is that a process, similar to aerobic protection against radicals, should occur in the complete absence of $\mathrm{O}_{2}$ and probably plays a significant role also in the presence of $\mathrm{O}_{2}$. With the decrease in $\left[\mathrm{O}_{2}\right]$ the anaerobic system of antiradical protection should progressively predominate. Although the radicals formed from sinks such as GSH and ascorbate may be less damaging than the first-generation radicals, the problem of how they disappear is of significance. Consider first that the only way they could disappear is by radical:radical reactions such as disproportionation or dimerization. In order for the sinks to be maintained by enzymes such as glutathione reductase, their radicals first need to be converted to the fully oxidized form. At a steady state and constant flux of dangerous radicals, radical:radical reactions will be significant only after significant portions of the sinks are converted to radicals. Furthermore, although the rate constants of some radical:radical reactions are high, they are second-order reactions; hence, they will become more significant at high concentrations of the radicals. It is important to keep in mind that the rates of reactions of a given radical with targets or with itself are determined not just by the second-order rate constant $k$ but also by the (pseudo) first-order rate constant k'. Thus, in the equation $\mathrm{v}=\mathrm{k}$ '[radical], $\mathrm{k}$ ' is equal to $\mathrm{k}$ [target] where 'target' could be a nonradical species, the same radical, or another radical. Hence, the existence of first-order pathways (with respect to the radical), such as catalysis of the radical:radical reactions or 1-electron reduction/oxida- tion of the radicals, will be a very effective way to dramatically decrease their concentrations. The catalyzed dismutation of $\mathrm{O}_{\dot{2}}^{\overline{2}}$, by SOD for example, is first order with respect to $\mathrm{O}_{2}^{\overline{2}}$, unlike the second-order spontaneous dismutation of $\mathrm{O}_{\dot{2}}^{\overline{2}}$ [29]. Such pathways exist anaerobically, as exemplified by the following experiment of $\mathrm{Gu}$ and Imlay [30]. They chemically reduced a redox-cycling agent (paraquat) and, using inverted E. coli vesicles, observed that the radical rapidly disappeared in the presence of fumarate as a final acceptor, but not in its absence or in the absence of vesicles. Certainly, in vivo this pathway is likely to not be that direct, especially for more oxidizing radicals, and can only serve, as the authors discuss, as a way of recycling the radical to the toxic fully oxidized form of the redox-cycling agent. In vivo, the first-generation radicals should first react mostly with other sensitive targets and radical sinks and the resulting radicals will be those that are oxidized or reduced directly or indirectly by the components of the respiratory chain. In this view, toxicity is actually due both to the radicals and to the depletion of cellular reductants such as NADPH, necessary for the replenishment of the sinks.

Ascorbate radical reductase activity and enzymes associated with it have been reported for a number of species [31]. Ascorbate radical reductase activity is a case of SROR activity. Are there radical:substrate oxidoreductases? Since the radical mentioned in the experiment of $\mathrm{Gu}$ and Imlay [30] was the highly reducing paraquat radical, this seems likely. Similarly, NAD rapidly reduces oxidized cytochrome $\mathrm{c}$ [10].

Are there enzymes or other redox factors capable of catalysis of radical:radical electron transfer (disproportionation) (RRORs)? Again, that is likely since even $\mathrm{Cu}$ and $\mathrm{ZnSOD}$, whose active center is well hidden, can react, albeit very slowly, with some redox couples, as ferri/ferro cyanide for example, using superoxide as an electron donor or acceptor, respectively [32]. Although none of the members of that couple are formally free radicals or are at least not usually thought of as such, these actions of SOD are a good analogy to SROR and RROR actions. In the cases of other enzymes or factors, the redox active metal or other component is not that well hidden. Hence, on many occasions potentially dangerous radicals and especially the radicals of the sinks are eliminated in the cell by being treated as metabolites and dealt with simply by metabolizing them back to the nontoxic fully reduced or oxidized forms. 


\section{Epilogue}

Halliwell [33] has discussed some problems concerning the use of cell cultures for studying the toxicity of free radicals and ROS as well as redox signaling. In part, as he points out, cultured cells are exposed to at least 10 times more oxygen than cells in organisms and therefore are in a state of oxidative stress even before the addition of $\mathrm{H}_{2} \mathrm{O}_{2}$ or other agents causing oxidative stress. As follows from the discussion above, the mechanisms of the production and disappearance of free radicals also depend on the concentration of oxygen.

In his recently published seminal review in Medical Principles and Practice entitled 'Oxygen: how do we stand it?' Fridovich [34] discussed the dangers imposed by the appearance of oxygen in the earth's atmosphere, such as generation of superoxide and other ROS. He also discussed the ingenious ways that evolved in order to protect cells from the toxicity of such agents.

In the present review, the focus is on oxygen-independent formation of free radicals and ways of eliminating such radicals. Primitive organisms were challenged by free radicals before the gaseous molecular oxygen appeared and evolved systems for the elimination of these radicals. After the appearance of oxygen, the oxygen-independent pathways of free radical formation and elimination did not (completely) disappear. Rather, the anaerobic protective systems evolved to work in cooperation with the aerobic systems such as superoxide dismutases, peroxidases, etc. Certainly, the views in this review are not in conflict but are rather in cooperation with the views expressed by Fridovich [34].

\section{Postscript: Radical Metabolism}

A large body of literature describes numerous beneficial effects exerted by antioxidants. Antioxidants, including glutathione, nutritional antioxidants such as ascorbate and vitamin E, phytochemicals, urate, vitamin A, etc., are an extremely versatile group of compounds. Traditionally, their beneficial effects were thought to be largely due to the ability of these compounds to scavenge harmful free radicals and ROS. Various mimics of superoxide dismutase and of other enzymes capable of metabolizing superoxide, $\mathrm{H}_{2} \mathrm{O}_{2}$, and other ROS are also thought to protect by metabolizing these harmful species into less harmful or benign species. A significant change in that view is occurring, however, especially in recent years. Thus, many reports now claim that low doses of phyto-

Free Radicals: How Are They

Metabolized? chemicals and SOD mimics exert beneficial effects by enforcing adaptations, including by acting as pro-oxidants or by means other than scavenging radicals.

While this review was being processed, two articles $[35,36]$ appeared which presented strong arguments that phytochemicals, other micronutrients and SOD and SOR mimics cannot possibly exert their beneficial effects by scavenging free radicals such as hydroxyl radicals, carbonate radicals, superoxide, and others. An important argument of the authors of these two papers is that the intracellular concentration of these agents is much smaller than the concentration of the other targets for free radicals in the cell. Indeed, these targets include amino acids, bases, sugars, lipids, and others, which are constituents of cellular components such as proteins, nucleic acids, and structures like biomembranes, and SODs and other sinks for superoxide. At the same time, the rate constants for the reactions of the alleged radical scavengers with radicals such as hydroxyl and carbonate radicals and superoxide are not higher and in many cases are lower than the rate constants for the reaction of these radicals with their targets.

However, I still maintain that a number of endogenous and nutritional antioxidants such as GSH, ascorbate, vitamin E, and likely others, including urate and vitamin A, exert beneficial effects in large part by acting as old-fashioned antioxidants-scavengers of free radicals, and in fact they act as catalytic antioxidants [35, 37]. On the other hand, Forman et al. $[36,38]$ expressed the view that only vitamin $\mathrm{E}$ acts as a radical scavenger and even joked that glutathione might be a 'sinker' rather than a radical sink. I think that the view of Forman et al. [36, 38] contradicts a large body of experimental evidence and theoretical thinking and therefore their too-radical view might discourage promising research. Even the total sum of the concentrations of ascorbate and glutathione plus other reduced compounds such as NADPH and urate is not enough to completely or even sufficiently protect other targets from the onslaught of extremely reactive radicals such as hydroxyl and carbonate radicals. Still, there will be some protection and these antioxidants should exert even greater protection against less reactive radicals. Nevertheless, as a consequence of the attack of reactive radicals, there will be substantial damage to proteins, nucleic acids, lipids, and other components. Initially, this damage will be manifested as the formation of the radicals of proteins and other constituents. The question now is how these radicals will disappear. This problem can be elucidated by examining the experiments and kinetic analysis of Domazou et al. [39]. Thus, a protein radical cannot 
disappear by a reaction with another component, such as another protein, since the 'old' radical will disappear but a new radical will be born. One possibility is for the protein radical $\left(\mathrm{P}^{\cdot}\right)$ to react with oxygen, and the resulting $\mathrm{POO}{ }^{*}$ radical might react with another protein. In this case, it is even worse since not only will a new protein radical appear but the initial protein will become damaged because the 1-electron reduction of $\mathrm{POO}^{*}$ (plus a proton) will lead to the formation of a nonradical protein peroxide $(\mathrm{POOH})$. There could be a way to repair $\mathrm{POOH}$ back to an intact protein, but this is not the main point being made here. According to these authors [39], reactions of ascorbate with radicals such as $\mathrm{P}^{\circ}$ and $\mathrm{POO}^{\circ}$ may account for the depletion of ascorbate observed during oxidative stress. Fortunately, in many cases ascorbate competes with oxygen for $\mathrm{P}^{*}$ and $\mathrm{P}^{*}$ will be converted back to the intact protein. Protein radicals are also efficiently repaired by urate and urate radicals can be reduced by ascorbate [40]. Clearly, when one contemplates the fate of $\mathrm{P}^{\cdot}$, one must consider that ascorbate is not the only antioxidant and that the reduction of $\mathrm{P}^{\cdot}$ and therefore the repair of the damaged protein will depend on the sum of the concentrations of the individual antioxidants (in a given compartment) multiplied by the rate constants of their reactions with $\mathrm{P}^{\cdot}$.

In this way, ascorbate, glutathione, and other reductants accomplish repair by acting as radical scavengers even when the scavenging of the initial radical results in damage, as is the case of the reaction of the $\mathrm{POO}^{\circ}$ radical with ascorbate, which leads to the production of $\mathrm{POOH}$, yet even in this case ascorbate acts as a chain breaker. Therefore, they are sinks in complete agreement with the radical sink hypothesis of Winterbourne [23]. The next question is what happens with the radicals of ascorbate, glutathione, NADPH, and other such scavengers. Ac- cording to the classical radical sink hypothesis, some of these radicals can directly react with $\mathrm{O}_{2}$, producing superoxide; that is the case of NADP', for example, while in the case of the glutathione radical the pathway leading to superoxide is more complex, as discussed above. Finally, SOD dismutes superoxide to nonradical products, while $\mathrm{NAD}^{+}$and GSSG are reduced back to GSH and NADPH by enzymes such as glutathione reductase and glucose6-phosphate dehydrogenase. However, the radical products of some scavengers like the ascorbyl radical do not react rapidly with oxygen and appear to be eliminated by enzymes such as ascorbate (ascorbyl)-radical reductase and likely, as I speculate in this review, by other enzymes capable of metabolizing the radicals formed during the oxidation of a small number of antioxidants. Thus, these antioxidants act as catalytic antioxidants (scavengers). The description by Forman et al. [36] of the scavenging of the radical formed during the oxidation of vitamin $\mathrm{E}$ by ascorbate, followed by reduction of the ascorbyl radical by the ascorbate radical reductase, is a case in which both vitamin $\mathrm{E}$ and ascorbate act as catalytic antioxidants as well. In contrast, the concentration of pharmaceutical phytochemicals and SOD mimics in the cell on which they have been reported to exert beneficial effects is orders of magnitude lower than that of glutathione and of the other catalytic antioxidants, and hence the mimics and the phytochemicals cannot possibly exert their effects by acting as radical scavengers.

\section{Acknowledgements}

The author is thankful to Dr. I. Fridovich for helpful discussions and constructive criticism.

\section{References}

1 Tarpey MM, Fridovich I: Methods of detection of vascular reactive species: nitric oxide, superoxide, hydrogen peroxide and peroxynitrite. Circ Res 2001;89:224-236.

2 Murphy MP, Holmgren A, Larsson NG, et al: Unraveling the biological roles of reactive oxygen species. Cell Metab 2001;13:361-364.

-3 Kalyanaraman B, Darley-Usmar V, Davies KJA, et al: Measuring reactive oxygen and nitrogen species with fluorescent probes: challenges and limitations. Free Radic Biol Med 2012;52:1-6.
4 Rota C, Fann YC, Mason RP: Phenoxyl free radical formation during the oxidation of the fluorescent dye $2^{\prime}, 7^{\prime}$-dichlorofluorescein by horseradish peroxidase: possible consequences for oxidative stress measurements. J Biol Chem 1999;274: 28161-28168.

5 Dutton DR, Reed GA, Parkinson A: Redox cycling of resorufin catalyzed by rat liver microsomal NADPH-cytochrome P450 reductase. Arch Biochem Biophys 1989;268:605-616.

6 Zhao B, Ranguelova K, Jiang J, et al: Studies on the photosensitized reduction of resorufin and implications for the detection of oxidative stress with Amplex Red. Free Radic Biol Med 2011;51:153-159.
7 Buettner GR, Ng CF, Wang W, et al: A new paradigm: manganese superoxide dismutase influences the production of $\mathrm{H}_{2} \mathrm{O}_{2}$ in cells and thereby their biological state. Free Radic Biol Med 2006;41:1338-1350.

$\checkmark 8$ Bielski BH, Chan PC: Re-evaluation of the kinetics of lactate dehydrogenase-catalyzed chain oxidation of nicotinamide adenine dinucleotide by superoxide radicals in the presence of ethylenediaminetetraacetate. J Biol Chem 1976;251:3841-3844. 
-9 Liochev SI, Fridovich I: Superoxide generated by glutathione reductase initiates a vanadatedependent free radical chain oxidation of NADH. Arch Biochem Biophys 1992;294: 403-436.

10 Avigliano L, Carelli V, Casini A, et al: Oxidation of nicotinamide coenzyme dimers by one-electron-accepting proteins. Biochem 1986;237:919-922.

11 Bielski BH, Chan PC: Kinetic study by pulse radiolysis of the lactate dehydrogenase-catalyzed chain oxidation of nicotinamide adenine dinucleotide by $\mathrm{HO}_{2}$ and $\mathrm{O}_{2}^{-}$radicals. J Biol Chem 1975;250:318-321.

12 Song Y, Buettner GR: Thermodynamic and kinetic considerations for the reaction of semiquinone radicals to form superoxide and hydrogen peroxide. Free Radic Biol Med 2010;49:919-962.

13 Liochev SI, Fridovich I: Lucigenin (bis-Nmethylacridinium) as a mediator of superoxide anion production. Arch Biochem Biophys 1997;337:115-120.

14 Liochev SI, Fridovich I: Lucigenin luminescence as a measure of intracellular superoxide dismutase activity in Escherichia coli. Proc Natl Acad Sci USA 1997;94:2891-2896.

15 Liochev SI, Fridovich I: A cationic manganic porphyrin inhibits uptake of paraquat by Escherichia coli. Arch Biochem Biophys 1995; 321:271-275

-16 Imlay JA: Cellular defenses against superoxide and hydrogen peroxide. Annu Rev Biochem 2008;277:755-776.

17 Liochev SI, Benov L, Touati D, et al: Induction of the soxrs regulon of Escherichia coli by superoxide. J Biol Chem 1999;274:9479-9481.

18 Liochev SI, Fridovich I: A mechanism for complementation of the sodA sodB defect in Escherichia coli by overproduction of the rbo gene product (desulfoferrodoxin) from $\mathrm{De}$ sulfoarculus baarsii. J Biol Chem 1997;272: 25573-25575.
19 Van Raamsdonk JM, Hekimi S: Superoxide dismutase is dispensable for normal animal lifespan. Proc Natl Acad Sci USA 2012;109: $5785-5790$

20 Ansenberger-Fricano K, Ganini D, Mao M, et al: The peroxidase activity of mitochondrial superoxide dismutase (MnSOD/SOD2). Free Radic Biol Med 2013;54:116-124.

21 Liochev SI, Fridovich I: Peroxidase activity by MnSOD? Free Radic Biol Med 2012, E-pub ahead of print.

22 Mason RP: Two hypotheses for the peroxidase activity of Mn-superoxide dismutase Free Radic Biol Med 2013, E-pub ahead of print.

23 Winterbourn CC: Reconciling the chemistry and biology of reactive oxygen species. Nat Chem Biol 2008;4:278-286.

24 Imlay JA: What biological purpose is served by superoxide reductase? J Biol Inorg Chem 2002;7:659-663.

25 Lombard M, Fontecave M, Touati D, et al: Reaction of the desulfoferrodoxin from Desulfoarculus baarsii with superoxide anion: evidence for a superoxide reductase activity. J Biol Chem 2000;275:115-121.

26 Coulter EDA, Kurtz DM Jr: Role for rubredoxin in oxidative stress protection in Desulfovibrio vulgaris: catalytic electron transfer to rubrerythrin and two-iron superoxide reductase. Arch Biochem Biophys 2001;394:76-86.

27 Rodrigues JV, Gomes CM: Enhanced superoxide and hydrogen peroxide detection in biological assays. Free Radic Biol Med 2010;49: 61-66.

28 Wardman P: Evaluation of the 'radical sink' hypothesis from a chemical-kinetic viewpoint. J Radioanalyt Nuclear Chem 1998;232: 23-27.
29 Fridovich I: Superoxide dismutases an adaptation to a paramagnetic gas. J Biol Chem 1989;264:7761-7764.

30 Gu M, Imlay JA: The SoxRS response of Escherichia coli is directly activated by redox-cycling drugs rather than by superoxide. Mol Microbiol 2011;79:1136-1150.

- 31 Yu S-W, Kim YR, Kang SO: Spectral characterization and chemical modification of FMN-containing ascorbyl free-radical reductase from Pleurotus ostreatus. Biochem J 1999; 341:755-763.

32 Liochev SI, Fridovich I: Copper- and zinccontaining superoxide dismutase can act as a superoxide reductase and a superoxide oxidase. J Biol Chem 2000;275:38482-38485.

33 Halliwell B: Free radicals and antioxidants quo vadis? Trends Pharmacol Sci 2011;30: 125-130.

34 Fridovich I: Oxygen: how do we stand it? Med Princ Pract 2013;22:131-137.

35 Liochev SI: Superoxide dismutase mimics, other mimics, antioxidants, prooxidants and related matters. Chem Res Toxicol 2013;26: 1312-1319.

36 Forman HJ, Davies KJ, Ursini F: How do nutritional antioxidants really work: nucleophilic tone and para-hormesis versus free radical scavenging in vivo. Free Radic Biol Med 2013, E-pub ahead of print.

37 Liochev SI: Reactive oxygen species and the free radical theory of aging. Free Radic Biol Med 2013;60:1-4.

38 Forman HJ, Traber M, Ursini F: Antioxidants: GRABbing new headlines. Free Radic Biol Med 2013, E-pub ahead of print.

39 Domazou AS, Zelenay V, Koppenol WH, et al: Efficient depletion of ascorbate by amino acid and protein radicals under oxidative stress. Free Radic Biol Med 2012;53:15651573.

40 Domazou AS, Zhu H, Koppenol WH: Fast repair of protein radicals by urate. Free Radic Biol Med 2012;52:1929-1936.
Free Radicals: How Are They Metabolized?
Med Princ Pract 2014;23:195-203 